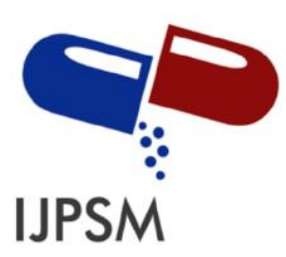

Georgiana Farrugia Bonnici et al, Int. Journal of Pharmaceutical Sciences and Medicine (IJPSM),

Vol.6 Issue. 8, August- 2021, pg. 1-16

ISSN: 2519-9889

Impact Factor: 3.426

\title{
Are Maltese General Practitioners' Musculoskeletal Imaging Requests Indicated for Diagnostic or Clinical Management Purposes? - A Research Study
}

\author{
Georgiana Farrugia Bonnici ${ }^{1}$; Ryan Grech ${ }^{2}$ \\ ${ }^{1}$ Department of Family Medicine, Faculty of Medicine \& Surgery, University of Malta, gfar0010@um.edu.mt \\ ${ }^{2}$ Department of Radiology, NHS Greater Glasgow \& Clyde, ryan.grech@ggc.scot.nhs.uk
}

DOI: 10.47760/ijpsm.2021.v06i08.001

\begin{abstract}
Research Objectives: This research study aimed to determine whether the patients' exposures to ionizing radiation via plain musculoskeletal radiography are indicated for the diagnostic or clinical management purposes, according to standards defined in the European Commission (Radiation safety 118) Referral Guidelines for Imaging (2001), as well as the Royal College of Radiologists (RCR) iRefer guidelines (2017).

Research Methods: A non-experimental, exploratory research design was adopted to answer the objectives of this study. Data were collected through 694 electronic, musculoskeletal imaging requests that were selected via cluster random sampling. The data collected were quantitative in nature. The study had a $3.7 \%$ margin of error (at a confidence level of $95 \%$ ) in relation to the analysed imaging request forms. Data were analysed using descriptive statistics. A p-value of $\leq 0.05$ at a confidence level of $95 \%$ was considered as statistically significant.

Results: Only $20.5 \%$ of plain, musculoskeletal imaging requests that are being referred by Maltese, state health centres G.Ps are classified as indicated for diagnostic or clinical management purposes. In addition, $65.3 \%$ of plain, musculoskeletal imaging requests were classified in the not routinely indicated category. The lumbo-sacral spine is the region that has registered the highest number of cases in the not routinely indicated category; being closely followed by the knee and the cervical spine respectively. These findings indicate that there is a greater tendency for Maltese state health centres G.Ps to request plain, musculoskeletal imaging investigations which are not routinely warranted according to the European Commission RP118 (2001) or the RCR i-Refer (2017) gold-standard imaging referral guidelines. Moreover, $40.0 \%$ of these imaging requests were also referred with insufficient clinical details.

Recommendations: Based on the research findings as well as the available literature, the following recommendations are being suggested: Continuing medical education in radiation safety and imaging referral criteria for Maltese state health centres G.Ps and G.P. trainees; Issuing regular departmental memos about any related evidence-based research findings; Ensuring the availability of the imaging referral guidelines on the desktops of all workplace computers, and promoting the use of imaging referral guidelines as well as kindling the safetynet of asking for advice from senior colleagues or radiologists whenever the referrers are in doubt about the management of a particular clinical case; Promoting the development of refinements and updates of locally applicable imaging referral guidelines to address any potential gaps in the older versions; Considering the introduction of a pro-forma clinical checklist for requesting online plain imaging investigations to aid G.Ps in improving the appropriateness of their imaging requests at times of clinical controversy, as well as in providing all the relevant clinical details for reporting; Organizing a radiation protection campaign to both the Primary Health Care Department (Malta) as well as to members of the general public and considering the use of radiation protection booklets or posters that may be distributed or show-cased to the patients whilst still in the primary care clinics waiting areas.
\end{abstract}

Keywords: family medicine, general practitioners, imaging, indication, Musculo-skeletal 


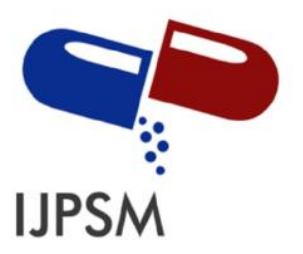

Georgiana Farrugia Bonnici et al, Int. Journal of Pharmaceutical Sciences and Medicine (IJPSM),

Vol.6 Issue. 8, August- 2021, pg. 1-16

ISSN: 2519-9889

Impact Factor: 3.426

\section{1 - Introduction}

The European Commission (2001) recognizes that the most common causes of the inefficient use of radiological resources are primarily coming from repeating imaging investigations which have already been done, requesting investigations when the imaging results are unlikely to affect the patients' diagnoses or clinical management, investigating too often, requesting the wrong investigations, as well as failing to provide the appropriate clinical information that the requested imaging investigations should answer. Unfortunately, most of the available studies that analyse the over-use of radiological investigations have mainly focused on high-radiation dose imaging techniques, such as CT (Almén, et al., 2009; Lehnert and Bree, 2010). However, these techniques account for only up to $20 \%$ of all imaging tests that are routinely carried out in clinical practice (Malone, et al., 2012; Zontar, et al., 2015). Conversely, plain x-rays have been less studied in relation to their over-use, despite amounting to over 70 $\%$ of the total imaging referrals (Malone, et al., 2012; Zontar, et al., 2015).

Thus, in this study, the authors sought to investigate what is currently happening with the clinical indications of imaging referring within the setting of primary care in Malta, Europe. Nevertheless, as the term implies, imaging referral guidelines are not an absolute constraint on clinical practice but rather an evidence-based approach to promoting good medical practice, especially in those areas commonly causing clinical uncertainty or controversy (European Commission, 2001; RCR, 2017).

\section{1 - Research Methodology}

\subsection{1 - Research design}

A non-experimental, exploratory research design was adopted to answer the objectives of this study, which has primarily aimed to determine whether the patients' exposures to ionizing radiation via plain musculoskeletal radiography are indicated for the diagnostic or clinical management purposes, according to standards defined in the European Commission (Radiation safety 118) Referral Guidelines for Imaging (2001), as well as the Royal College of Radiologists (RCR) i-Refer guidelines (2017).

\subsubsection{1 - The target population for the selection of the imaging referrals}

The eligibility criteria for inclusion in the sampling pertaining to the retrospective selection of the electronic, imaging request forms have consisted of musculoskeletal system plain $\mathrm{x}$-ray referrals which have satisfied all of the requirements listed below:

- Lower Limb Radiography: Knee and Hip;

- Upper Limb Radiography: Shoulder;

- Spinal Radiography: Cervical and Lumbo-sacral spine;

- Male and female adults above 18 years of age;

- Requested electronically via iCM ${ }^{\mathrm{TM}}$ (iClinical Manager ${ }^{\mathrm{TM}}$ ) by a G.P. from any of the 10 local, state health centres in Malta and Gozo (Gzira, Floriana, Qormi, Paola, Cospicua, Mosta, Rabat, Birkirkara, Kirkop, Victoria);

- Retrospectively over a period of one month, in August 2019.

Exclusion criteria have included those electronic, musculoskeletal plain imaging requests that were requested by G.Ps for paediatric patients under 18 years of age, as both gold-standard guidelines cater for fewer paediatric musculoskeletal imaging indications. All electronic, musculoskeletal imaging requests pertaining to the non-ionizing radiation modalities, namely MRI and US were excluded as these were outside the scope of this study. 


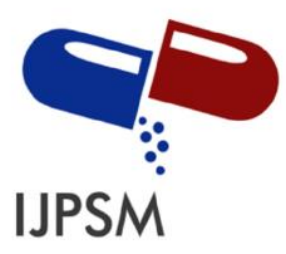

Georgiana Farrugia Bonnici et al, Int. Journal of Pharmaceutical Sciences and Medicine (IJPSM), Vol.6 Issue. 8, August- 2021, pg. 1-16

ISSN: 2519-9889

Impact Factor: 3.426

\subsubsection{2 -Random cluster sampling for the selection of the imaging referrals}

Random cluster sampling was used to select a retrospective sample of electronic imaging referrals for this study. A priori-power analysis has determined that a sample of approximately 700 imaging referrals from a very large population will guarantee a representative sample, with a desired narrow margin of error of $3.7 \%$, assuming a confidence level of $95 \%$ and a level of significance of 0.05 (Creative Research Systems, 2020; Camilleri, 2020).

Thereby, the sample utilized for this study consisted of 694 musculo-skeletal imaging requests that were requested electronically by various health centre G.P.s over a period of one month. In order to draw a sample which is as representative as possible of the target population, the author have included all the available samples that have matched the pre-defined eligibility criteria which were retrospectively requested in August 2019. Thus, for each subcategory of the musculo-skeletal imaging requests, the cluster sample that has been utilized in this study has included a total of 240 plain knee x-ray requests, 64 plain hip x-ray requests, 145 plain shoulder $x$-ray requests, 102 plain cervical spine $x$-ray requests, and 143 plain lumbo-sacral spine $x$-ray requests. Such electronic, imaging requests were drawn by the local, state hospital data intermediary via the SQL software because this electronic process helps in reducing the sampling bias by eliminating any human errors that are associated with the manual, complex processes of randomisation.

\subsection{2 - Data collection processes}

\subsubsection{1 - The use of gold-standard, imaging referral guidelines}

The Radiation safety 118 (RP118) imaging referral guidelines (European Commission, 2001) were used by the authors as the primary gold-standard guidelines when analysing the appropriateness behind the clinical or the diagnostic indications of several musculoskeletal imaging requests. These guidelines, which are also acknowledged by the Royal College of Radiologists, bear the advantage of having a mixed-development methodology which is both expertderived and endorsed by respected authorities, as well as evidence-based from higher levels of evidence sources of literature, such as randomised controlled trials, meta-analyses, systematic reviews, as well as robust experimental or observational studies (European Commission, 2001). The RP118 imaging referral guidelines were preferred by the author over other non-European based guidelines primarily because this research study is based in Malta, which is an European Union member state. Such guidelines were in fact, developed and issued by the European Commission in 2001. As a result, these are the imaging referral guidelines which are officially used in the Maltese Islands. In fact, they are most readily available for free locally via the official government online health portals. Moreover, these guidelines cover a good range of musculoskeletal and trauma conditions which in turn have permitted the analysis of a greater spectrum of diseases, and invariably, limited the amount of the non-analysed request forms due to the omission of certain non-specific conditions in the gold-standard imaging referring guidelines.

Since the RP118 European Commission imaging referral guidelines (European Commission, 2001) have the disadvantage of not being recent, the authors have also chosen a secondary set of gold-standard guidelines, namely the U.K. Royal College of Radiologists (RCR) i-Refer imaging guidelines, which are very similar to the latter but were more recently established in 2017.

\subsubsection{2 - The development of the research tool}

A clinical checklist was developed by the authors in a layout that is similar to what is presented in the European Commission Radiation safety 118 referring guidelines for imaging (European Commission, 2001) and the Royal College of Radiologists (RCR) i-Refer guidelines (2017). To facilitate the process of data analysis, the clinical checklist contains space for inputting the clinical indications and the types of imaging tests that are being requested by the referring G.Ps. All the clinical indications that were listed by the G.Ps on the musculo-skeletal imaging request forms 


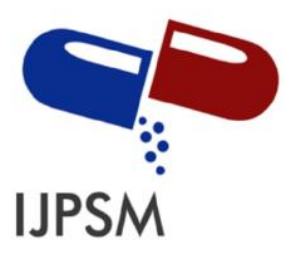

Georgiana Farrugia Bonnici et al, Int. Journal of Pharmaceutical Sciences and Medicine (IJPSM),

Vol.6 Issue. 8, August- 2021, pg. 1-16

ISSN: 2519-9889

Impact Factor: 3.426

were organized in this clinical checklist and were manually analysed according to the standards defined in the spine, the musculo-skeletal system and the trauma sections of the European Commission (Radiation safety 118) Referral Guidelines for Imaging (2001) and the RCR i-Refer guidelines (2017).

All the 694 clinical indications were classified by the two independent assessors in only one of the six categories listed below:

- Indicated: If the result of the imaging investigation will alter the patient's management or add confidence to the physician's diagnosis;

- Not indicated: If the supposed rationale for the requested investigation is untenable;

- Not indicated initially: If the clinical problem usually resolves with time, hence it would be advised deferring the investigation by approximately three to six weeks and only performing it then if the symptoms continue;

- Not routinely indicated: If the investigation is likely to be carried out only if a clinician gives cogent arguments for it;

- Specialized investigation: If the investigation is either complex or expensive and will usually be performed only after vetting it with a radiology specialist for those clinicians who have the relevant clinical expertise to evaluate the clinical findings and act on the imaging results;

- Not included in the guidelines: If the clinical indication on the imaging request forms did not match any criteria in both the European Commission RP118 and the RCR i-Refer imaging referral guidelines.

This clinical checklist has served as a clinically sound and straight-forward method to draw conclusions about the justification of the clinical indications of several imaging requests. A disadvantage to this clinical checklist is that there are strictly set-criteria for correct answers and thus, the findings may not contain any mid-way dimensions (Beck and Polit, 2010). An additional comments section was also added for every imaging request being analysed, in case more information needed to be expressed by the two assessors on any dubious cases.

In an attempt to increase the internal validity of the study, such a clinical checklist was filled in twice by the two authors of this study. The same samples of electronic imaging request forms were analysed by both assessors using a standardized approach which has ensured that all imaging request forms were being analysed in a fair and consistent manner. Such a standardized approach has demanded that all 694 imaging request forms are to be classified only in one category, namely as 'indicated', 'not indicated', 'not routinely indicated', 'not indicated initially', 'specialized investigation', or as 'not included in the guidelines' as per the primary, gold-standard, European Commission (2001) RP 118 imaging referral guidelines.

In those cases where the clinical indications written on the imaging request forms did not match with any of the referral criteria in the primary, gold-standard European Commission (2001) RP 118 imaging referral guidelines, the assessors had to also refer to the secondary, gold-standard, RCR i-Refer (2017) imaging referral guidelines. The two assessors have opted to classify an imaging request as 'not included in the guidelines', only if the clinical indications on the imaging request form did not match any referral criteria in both the European Commission RP118 and the RCR i-Refer imaging referral guidelines. Moreover, both assessors were required to ascertain complete objectivity in their assessments, by basing their classifications of all imaging request forms solely on those clinical indications as well as any other referral information that was written by the requesting physicians on their electronic, imaging request forms. No direct contacts with the referring physicians, the patients, or the patients' medical notes have been made throughout these assessments. In those very few cases, where the classifications of the imaging requests forms did not initially tally between the two assessors, a consensus was sought via an adequate discussion to settle any clinical disagreements. 


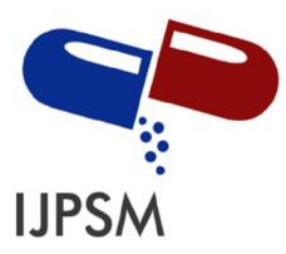

Georgiana Farrugia Bonnici et al, Int. Journal of Pharmaceutical Sciences and Medicine (IJPSM), Vol.6 Issue. 8, August- 2021, pg. 1-16

ISSN: 2519-9889

Impact Factor: 3.426

\subsubsection{3 - The validity and the reliability of the research tool}

Some degree of face validity for the clinical checklist was established by an academic supervisor who has assessed the clarity and the readability in both the formatting and the general layout of the clinical checklist and whether the research tool has addressed the objectives of this research study. Moreover, content-related validity was used to enhance the validity of research of the tool via assessing the adequacy and the relevance of all the elements that have been included in the clinical checklist. Three academic professors in radiography were asked to rate both the clarity and the relevance of all the elements in the clinical checklist. All of the elements in the clinical checklist were rated as very relevant and very clear by the three experts, hence producing an inter-rater Content Validity Index (CVI) of $100 \%$ for the clinical checklist.

The reliability of the research tool that have been used in this study was determined through stability reliability. With regards to the clinical checklist, both authors have analysed the same ten cases twice within one week, in order to establish whether the classifications of the clinical indications that have been inputted on the clinical checklist were the same. A favourable reliability coefficient value of 0.95 was obtained for the clinical checklist.

\subsubsection{4 - The pilot study}

The authors have analysed a set of twenty cases as a trial run in September 2019, in order to be able to test the standardized approach to the data classification prior to the actual study. The results of this pilot study were also discussed with a University of Malta statistician, in order to be able to conduct the priori-power analysis and plan the data analyses processes for the actual study (Camilleri, 2020).

\subsubsection{5 - Ethical considerations}

Permission to conduct this research study was sought and granted from the University of Malta Research Ethics Committee, as well as the Primary Health Care Department of Malta. Furthermore, the collection of the data pertaining to the imaging request forms was in line with an employee privacy policy of the PHCD (Malta), which states that the employees of this department may be subjected to participate in any form of audits that are periodically conducted to improve the clinical care as well as to safeguard the health of the patients.

G.P's were not faced with any moral, physical or emotional harm by participating in this research study. The rights to privacy and confidentiality were maintained at all stages of the research process, and the authors have explicitly stated that all the information that will be collected will be treated with confidentiality. Moreover, data was not revealed in such a way that individual participants could be identified. It was also made clear to the G.P. participants that all data will be stored in line with the European data protection act in a password-protected computer and that it will be destroyed after the successful completion of this research study. Moreover, in order to safeguard the anonymity measures, the G.Ps requesting the analysed imaging investigations were not known to the authors.

\subsubsection{6 - Data analysis}

The data collection of existing medical records via the SQL software was carried out through a data intermediary who was in direct communication with the authors. Specific inclusion criteria for the type of clinical data that needed to be extracted were delineated to fulfill the objectives of this research study. Data were collected retrospectively. Following coding, data were presented to the authors in a Microsoft Excel ${ }^{\circledR}$ sheet. The identities of the referring physicians and the patients could not be identified. Several bar graphs were compiled using MS Excel ${ }^{T M}$ to facilitate the presentation of the research findings. Data in this section were primarily analysed by means of descriptive statistics; namely via the frequencies, the percentages as well as the mode as a measure of central tendency, which 


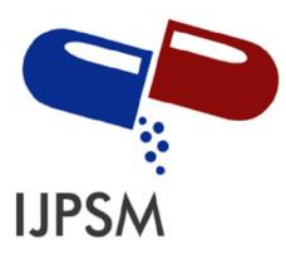

Georgiana Farrugia Bonnici et al, Int. Journal of Pharmaceutical Sciences and Medicine (IJPSM),

Vol.6 Issue. 8, August- 2021, pg. 1-16

ISSN: 2519-9889

Impact Factor: 3.426

have enabled the author to reveal the desired objectives and characteristics within the sampled, existing data-sets (Grove and Cipher, 2017; Camilleri, 2020).

\subsubsection{7 - The limitations of the research study}

The findings that have been generated from the analysis of the electronic imaging request forms exclude any data from Maltese private practice G.Ps, since the imaging referring system for the local G.Ps working in the private sector was mainly paper-based at the time of data collection. Thus, there is an under-representation of this particular cohort of Maltese G.P. referrers in this research study.

The analysis of the imaging request forms was based solely on the clinical indications that were written by the health-centre G.Ps on their electronic imaging referrals. In order to ascertain anonymity, the authors had no direct contact with neither the patients nor the referring physicians. Whilst a standardized approach to data analysis was established, minor assumptions about the patients' medical histories or their clinical well-being might have been made by these assessors. This in turn, could have generated potential human errors.

Despite being evidence-based, the gold-standard, imaging referring guidelines cannot cover all real-life clinical scenarios. Ultimately, these guidelines are there to guide the referring physicians but not to dictate the clinical management of their patients. Moreover, this research study's assessors may have been unaware of some other missing but rather relevant clinical data in the patients' medical histories that collectively could have merited the indications for more valid imaging requests. In fact, a very small amount of the analysed cases eventually had to be listed by the authors as not available in the guidelines, in order to avoid the skewing of results by having them misleadingly classified as indicated or not indicated.

\section{1 - Results and Discussion}

For the purpose of clarity, all percentage figures referred to in the findings of this research study have been rounded up to the nearest one decimal place.

\subsection{1 - The frequency distribution of the total indicated versus the non-indicated musculoskeletal imaging requests}

Out of 694 imaging requests, only 142 requests $(20.5 \%)$ were classified as indicated. The majority of these imaging requests ( $n=453,65.3 \%$ ) were classified in the not routinely indicated category (Refer to Figure 1 ). These findings indicate that there is a tendency for Maltese G.Ps. to request plain imaging investigations which are not always warranted according to the RP118 or the i-Refer gold-standard imaging referral guidelines.

Whilst no recommendations are absolute, such indications are generally classified as not routinely indicated because they should only be carried out if the referrers give cogent arguments for them (European Commission; 2001, RCR, 2017). A common clinical example of a non-routinely indicated evaluation via plain radiography in general practice would be of an elderly patient presenting with chronic low back pain, in whom there are no positive clinical findings to suggest an underlying serious condition. Thus, requesting a lumbo-sacral spine $x$-ray to evaluate only degenerative disease is not recommended as this will likely not improve the clinical outcomes. On the other hand, if this same request had to be done for the suspicion of a vertebral fracture, or pointers to metastases or infection, it then becomes indicated (European Commission; 2001, RCR 2017; Lim, et al., 2019). Nonetheless, spinal MRI remains the preferred modality of investigation for the diagnoses of most spinal conditions because it is helpful in identifying those cases which may benefit from planned surgical interventions or advanced pain management options (European Commission; 2001, RCR 2017). 


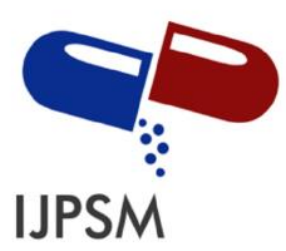

Georgiana Farrugia Bonnici et al, Int. Journal of Pharmaceutical Sciences and Medicine (IJPSM),

Vol.6 Issue. 8, August- 2021, pg. 1-16

ISSN: 2519-9889

Impact Factor: 3.426

\section{The frequency distribution of the total indicated versus non-indicated musculo-skeletal imaging requests}

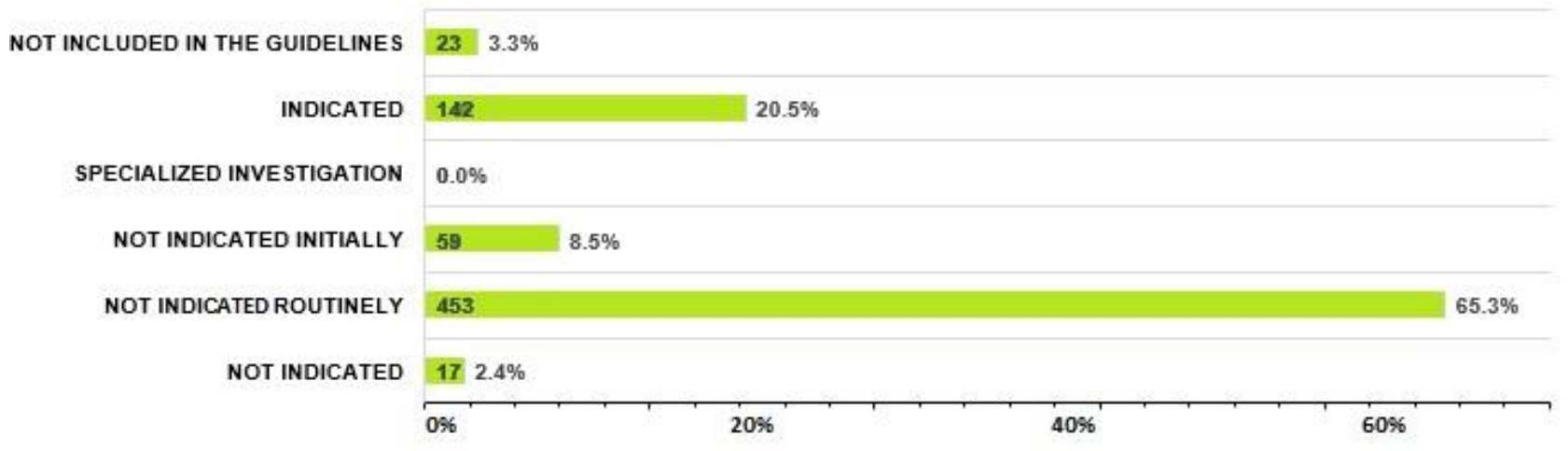

Figure 1 (The total indications of the imaging request forms)

The amount of non-indicated imaging requests in this study is slightly higher when compared to the findings that have been reported in the Spanish study by Vilar-Palop, et al., (2018), whereby almost half of the 2022 imaging requests being reviewed were deemed as appropriate (967, $47.8 \%$ ) and 728 imaging requests (36.0 \%) were considered as inappropriate. On the other hand, in another study by Rawle and Pighills (2018), results have similarly indicated that only a quarter ( $24.7 \%$ ) of the total imaging examinations have met the referring criteria for their local, diagnostic imaging pathways, while the remaining $75.3 \%$ of examinations have only partially met or did not meet the imaging referral criteria at all.

There are several reasons as to why these trends are being observed. Firstly, Mendelson and Montgomery (2016) and Azman, et al., (2019) argue that in a modern era coupled with refined technological advances, the perceived importance of the fundamental skills of history-taking and clinical examination may be declining. Moreover, time constraints and over-confidence in the accuracy of imaging modalities may also play a role in this cascade of overinvestigation (Mendelson and Montgomery, 2016; Lim, et al., 2019). There could also be a feeling among doctors that there is little practical action that can be taken to improve the situation; as well as communication failures between medical professionals over a prolonged period of time (Malone, et al., 2012). Furthermore, G.Ps are presumably more likely to be following up on the presence of chronic problems in the elderly, as opposed to the acute presentations in younger patients, which are often resolved in a shorter time span (Ashurst, et al., 2014; Lim, et al., 2019). Lastly, finding the right balance between imaging referring and patients' reassurance is vital, as iatrogenic damage from over-investigation may be a more common threat than legal proceedings (Hamada and Fujimichi, 2014; Mendelson and Montgomery, 2016; Brownlee, et al., 2017, Lumbreras, et al., 2018; Lim, et al., 2019).

Imaging referrals are the primary means of communication between the requesting physicians and the reporting radiologists. Therefore, it is vital that the imaging request forms are always presented for reporting with sufficient clinical information (European Commission, 2001; Pitman, 2017). There are various good quality attributes of valid imaging requests. Primarily, a referrer should take into account the patients' radiation safety as well as the attributable diagnostic or clinical management values of the requested imaging modalities (European Commission; 2001; RCR 2017; Pitman, 2017). Secondly, a clear clinical question that the referrers want to be answered must be 


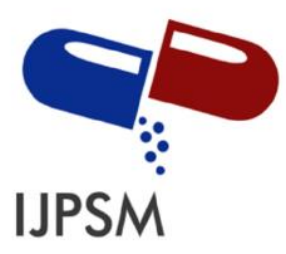

Georgiana Farrugia Bonnici et al, Int. Journal of Pharmaceutical Sciences and Medicine (IJPSM),

Vol.6 Issue. 8, August- 2021, pg. 1-16

ISSN: 2519-9889

Impact Factor: 3.426

formulated whilst keeping in mind that such information will also be assisting the reporting radiologists in the interpretation of the imaging results, as well as in the completion of pertinent imaging reports (European Commission; 2001; RCR 2017; Pitman, 2017). Such requests should always be written or typed in a legible, unambiguous and complete manner without any loss of content or meaning (European Commission; 2001; RCR 2017; Pitman, 2017). Sufficient demographic details as well as the contact details of both the patients and the referrers are also necessary for the reporting radiologists to be able to unequivocally confirm their identities. Moreover, the provision of these details will also favour unimpeded contact between the reporting radiologists, the referrers and the patients alike (Pitman, 2017).

It is notable that Maltese G.Ps in this study are leaving out important clinical details in their imaging requests $(n=278 ; 40.0 \%)$. The most common types of information that were omitted by the referrers include the patient's age, the joint's level of functional mobility as well as the onset and the duration of any reported musculoskeletal pain. Additionally, it is also possible that there is a greater element of ambiguity in the clinical terms that are used by local G.Ps in describing the diffuse signs and symptoms on their imaging referrals. Examples of these case scenarios would be those of knee pain or hip pain in which the referrer does not explicitly specify if the patient can weightbear. Another issue with the imaging requests pertaining to musculoskeletal pain is when the referrer does not quantify the term "long-standing", possibly without being aware that both the duration and the tolerance for pain are very subjective. To some patients, ten days may be as long-standing as three months. Another example would be in the case of a mechanical fall, with no other documented evidence of exquisite swelling or tenderness to the affected joint. Unfortunately, such non-specific criteria are unlikely to be directly catered for in the imaging referral guidelines which could in turn lead to an under-estimation of the percentage of appropriateness of the imaging referrals by G.Ps.

Vilar Palop, et al., (2018) have concluded that G.Ps were less likely to order appropriate imaging investigations compared to those physicians who were working in various hospital-based, clinical specialty departments. Specifically, $40.4 \%$ of the imaging referrals coming from G.Ps were considered inappropriate, whilst a lower rate of inappropriate imaging referrals 146 (18.9\%) was recorded from hospital clinical specialists. The findings from a previous local audit that was conducted by one of the authors of this study in 2016 also tally with the latter (Farrugia, 2016). In this audit, it was also concluded that out of 750 imaging requests by hospital physicians, 494 (66\%) were considered to be indicated for diagnostic or clinical management purposes (Farrugia, 2016).

A possible reason for these observations is that patients presenting in general practice were prone to have more diffuse symptoms as opposed to those visiting specialized clinics; which could in turn, instigate over-investigation (Vilar-Palop, et al., 2018). Hence, it is even more important for all the imaging request forms to be completed accurately and legibly by G.Ps in order to avoid any potential misinterpretation by the reporting radiologists, who in most straight-forward cases are likely to have no direct contact with either the patients or the referring doctors (European Commission, 2001; RCR, 2017). It has also been demonstrated that when the clinical details in the patients' medical records were reviewed in conjunction with their imaging referrals, the non-clinically indicated rates of examinations will be reduced (Rawle and Pighills, 2018). Nevertheless, in the case of general practice, this is not always possible since patients might be visiting different doctors for emerging complaints. In such cases, health information systems typically come in handy in reviewing a particular patient's profile; albeit there are usually access-control mechanisms in place that could also forbid the access to specified information to those referrers who are working outside the dedicated access zones (Rehani, 2017). 


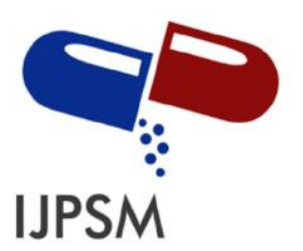

Georgiana Farrugia Bonnici et al, Int. Journal of Pharmaceutical Sciences and Medicine (IJPSM),

Vol.6 Issue. 8, August- 2021, pg. 1-16

ISSN: 2519-9889

Impact Factor: 3.426

\subsection{2 - The comparisons of the imaging indications by the types of body areas being investigated}

\subsubsection{1- Spinal imaging: The plain cervical and the lumbo-sacral spine requests}

Out of 102 plain cervical spine imaging requests, only $9.8 \%$ were classified as indicated. $83.3 \%$ of these imaging requests were classified in the not routinely indicated category (Refer to Figure 2). Comparingly, from 143 plain lumbo-sacral spine imaging requests, only $8.4 \%$ were classified as indicated. $88.1 \%$ of lumbo-sacral spine imaging requests were classified in the not routinely indicated category (Refer to Figure 3). These findings indicate that both the cervical spine and lumbo-sacral spine are two areas that are creating significant clinical controversy amongst Maltese G.Ps.

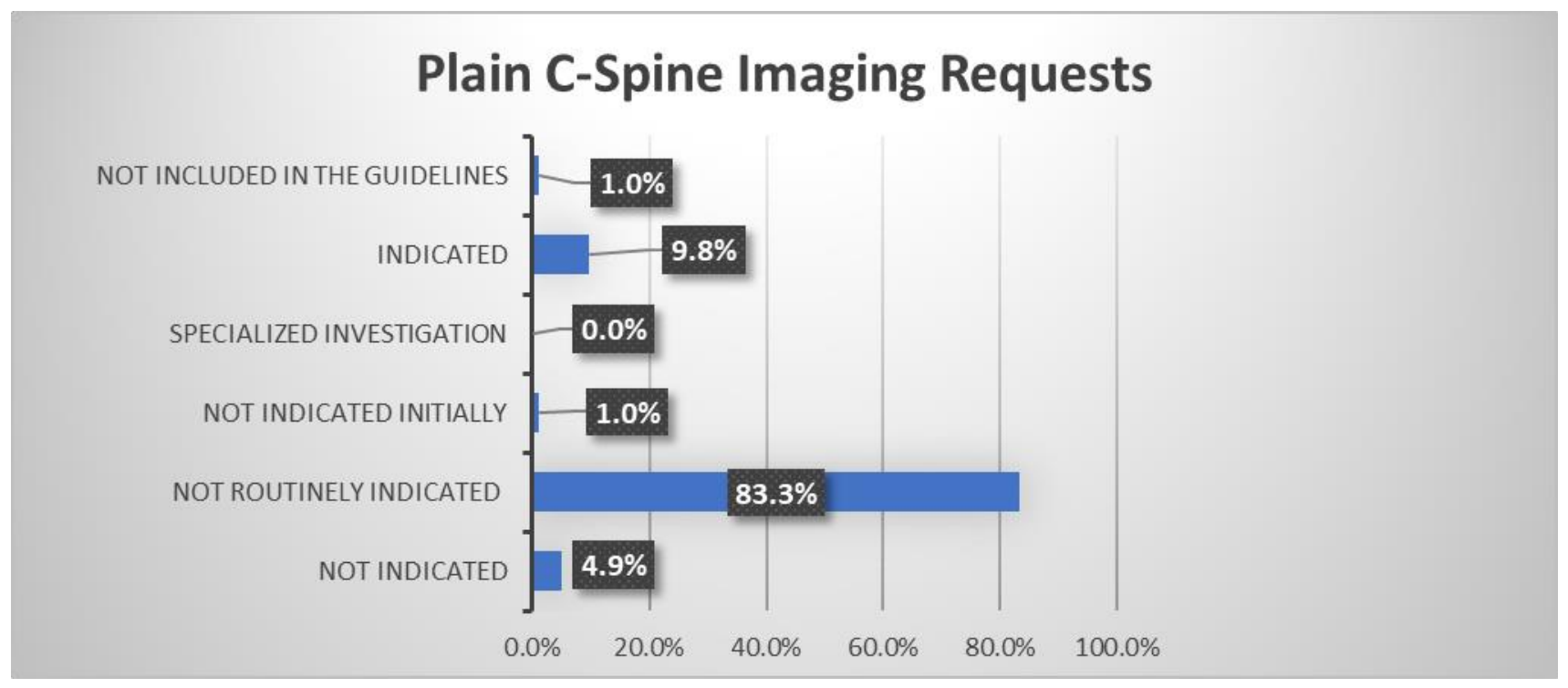

Figure 2 (The indications of the plain C-Spine imaging request forms)

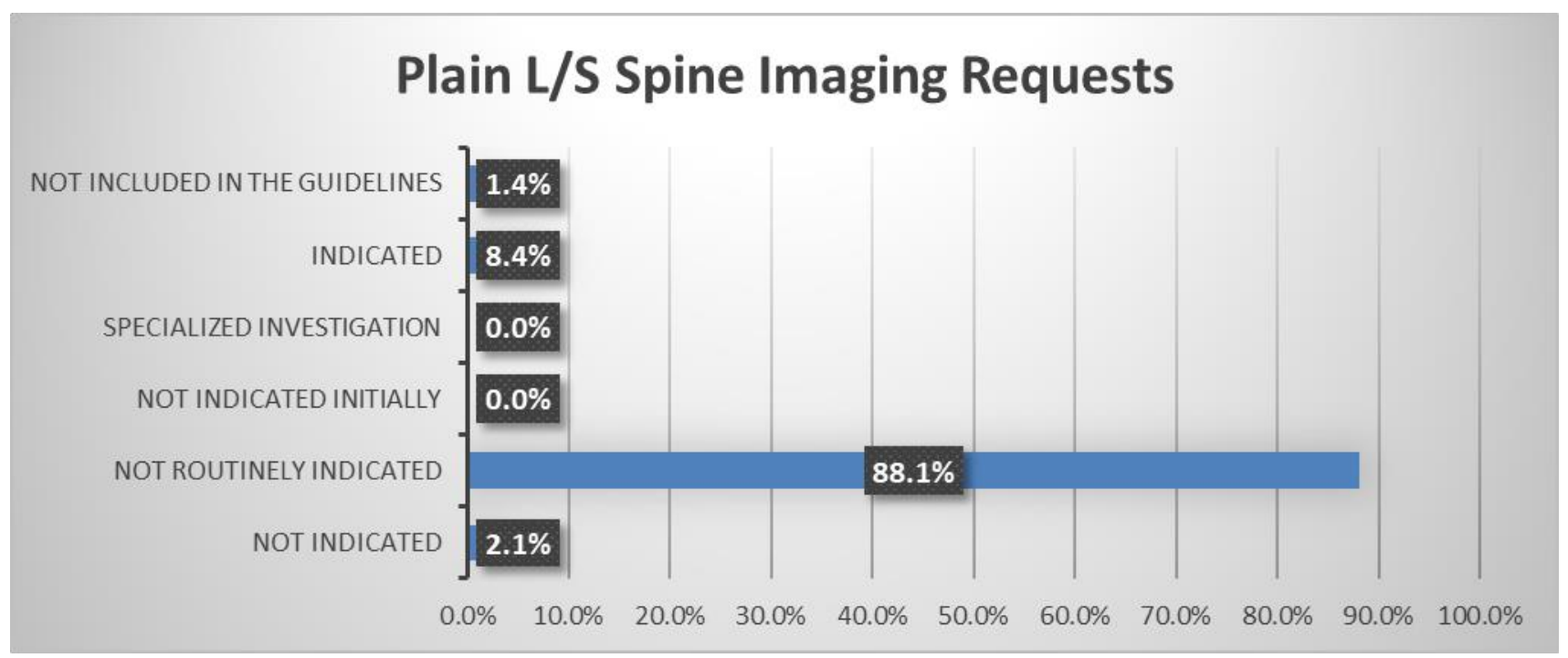

Figure 3 (The indications of the plain L/S Spine imaging request forms) 


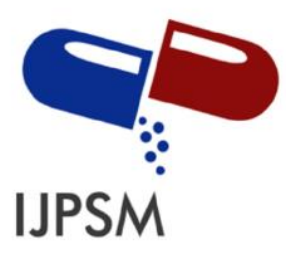

Georgiana Farrugia Bonnici et al, Int. Journal of Pharmaceutical Sciences and Medicine (IJPSM),

Vol.6 Issue. 8, August- 2021, pg. 1-16

ISSN: 2519-9889

Impact Factor: 3.426

Contrastingly, in another Maltese small-scale audit by Pullicino, et al., (2016), 64 out of 100 lumbo-sacral spine radiographs that were requested by medical doctors working in a central health centre, which were performed specifically for low back pain were indicated for such purposes. The authors of this study did not specify the speciality or the grades of the referrers. As in this study, it was noted that $35 \%$ of such lumbo-sacral spine radiographs were carried out with insufficient clinical details.

Non-specific, low back pain remains one of the most common musculo-skeletal conditions for which people seek medical advice (Suman, et al. 2018; Lim, et al., 2019). In fact, in the Netherlands, over $90 \%$ of patients have visited general practice clinics at least once per year regarding their low back pain (Suman, et al. 2018). Almost half of these patients were in turn referred for diagnostic imaging (Suman, et al. 2018). However, there is substantial evidence indicating that the diagnostic imaging that is requested for low back pain without any suggestion of a serious underlying cause does not improve the patient outcomes and can also pose significant radiation risks to these patients (European Commission, 2001; RCR 2017; Suman, et al. 2018, Lim, et al., 2019). The most common symptoms that are causing clinical controversy in the spinal categories amongst referrers in this research study are either neck pain or back pain, that could be managed conservatively as they were mostly attributable to various degenerative changes which begin in the early to middle ages and are often unrelatable to the presenting symptoms (European Commission; 2001, RCR 2017; Lim, et al., 2019). Such changes are often due to disc herniation or ligamentous changes, which are often undetectable on plain x-rays (European Commission, 2001; RCR 2017).

In the case of brachalgia which is significantly affecting the patient's lifestyle or in the presence of neurological deficits or other red-flag features such as vascular insufficiency, trauma, malignancy, infection, inflammation or myelopathy; MRI is more suitable as a primary method of investigation than plain radiography (European Commission, 2001; RCR 2017). Moreover, in the case of plain lumbo-sacral $x$-rays, their value may be greater in younger patients, particularly in the evaluation of primary bone tumours, spondylolisthesis or ankylosing spondylitis (European Commission, 2001; RCR 2017). On the other hand, plain radiography in elderly patients is more likely to be appropriate in characterizing the lesions in suspected cases of bone metastases, osteoporotic vertebral collapse or osteomyelitis (European Commission, 2001; RCR 2017).

\subsubsection{1 - Lower limb imaging: The plain hip and the knee requests}

From 64 plain hip imaging requests, only $10.9 \%$ were classified as indicated. In comparison to the cervical spine and lumbo-sacral spine categories, a slightly lower rate of $59.4 \%$ of imaging requests have been registered in the not routinely indicated category (Refer to Figure 4). On the other hand, the highest registered number of cases (17\%) in the 'Not included in the guidelines' category were also attributable to the hip. This is mostly in line with the insufficient clinical details that were being listed on the imaging request forms by G.Ps.

Moreover, from 240 plain knee imaging requests, only $13.3 \%$ were classified as indicated. It is notable that the knee has also registered the second highest numbers of fully indicated imaging requests by Maltese G.Ps, amongst all the five body regions that have been investigated in this study. This trend could be attributable to an increasing experience by G.Ps in dealing with various knee conditions; seeing that the knee was also the most commonly evaluated area by plain radiography within the cluster sample being analysed. Nonetheless, $85.0 \%$ of knee imaging requests were still classified in the not routinely indicated category (Refer to Figure 5). This indicates that the management of knee conditions may still potentially be problematic amongst local G.Ps.

Plain x-rays of the affected lower limb joints in a presenting arthropathy may be helpful in determining the cause, albeit bony erosions may be a relatively late feature (European Commission, 2001; RCR 2017). Contrastingly, in any 


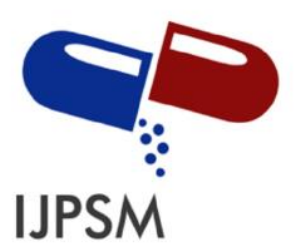

Georgiana Farrugia Bonnici et al, Int. Journal of Pharmaceutical Sciences and Medicine (IJPSM),

Vol.6 Issue. 8, August- 2021, pg. 1-16

ISSN: 2519-9889

Impact Factor: 3.426

follow-up arthropathy cases, plain x-rays are usually only needed by the orthopaedics or the rheumatology specialists who will ultimately be deciding on the clinical course of actions (European Commission, 2001; RCR 2017).

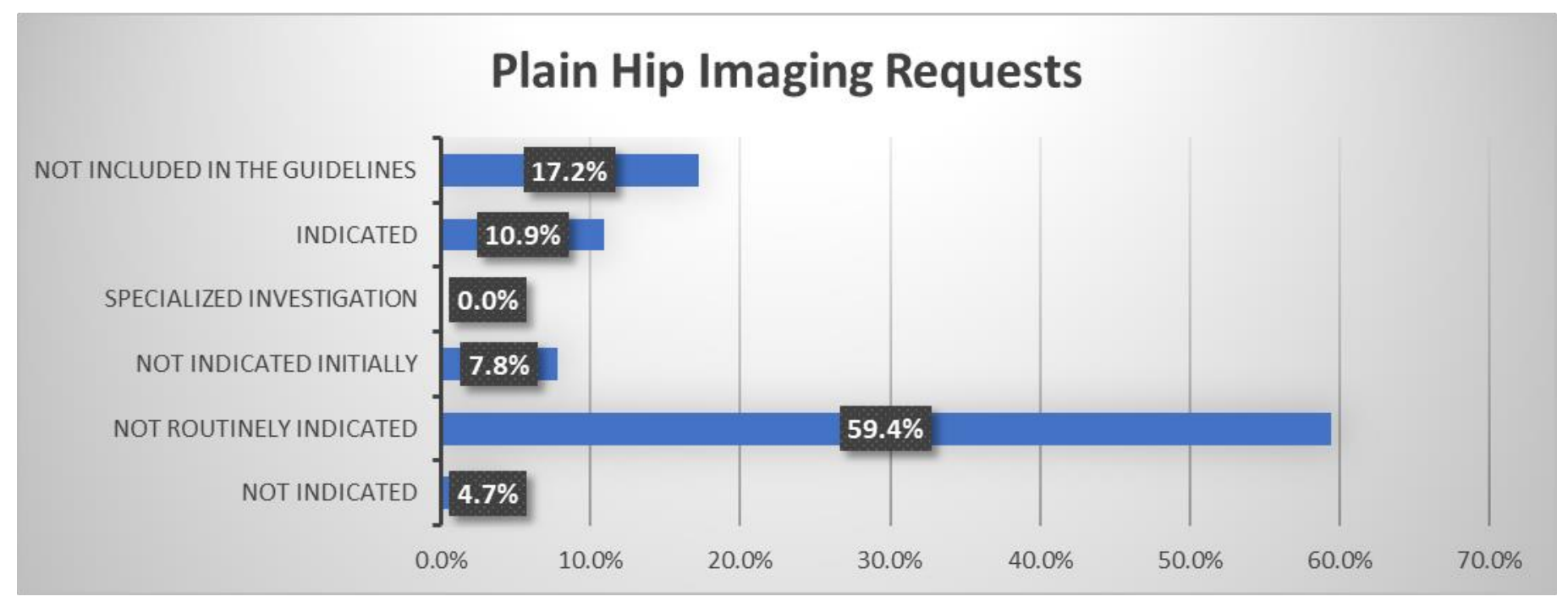

Figure 4 (The indications of the plain hip imaging request forms)

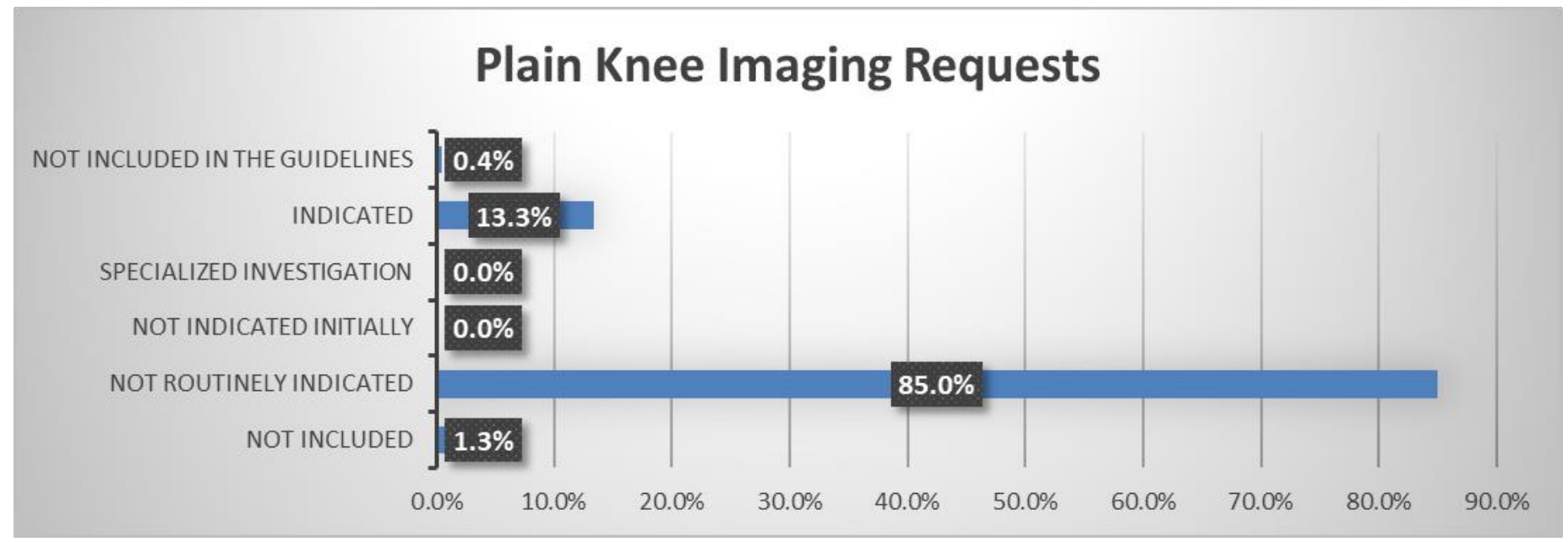

Figure 5 (The indications of the plain knee imaging request forms)

3.1.2.2 - Upper limb imaging: The plain shoulder requests

Out of 145 plain shoulder imaging requests, $36.5 \%$ were classified as not indicated initially. This classification includes those situations where the evidence shows that the clinical problem usually resolves with time. Therefore, it is recommended to defer the study by approximately three to six weeks and only perform $t$ then if the symptoms continue. Non-specific shoulder pain is a typical example, since degenerative changes in the acromio-clavicular joints and the rotator cuff are common (European Commission, 2001; RCR 2017). 


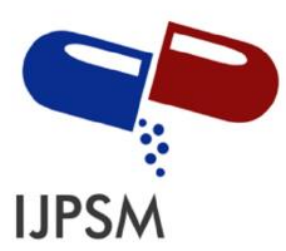

Georgiana Farrugia Bonnici et al, Int. Journal of Pharmaceutical Sciences and Medicine (IJPSM),

Vol.6 Issue. 8, August- 2021, pg. 1-16

ISSN: 2519-9889

Impact Factor: 3.426

Plain x-rays are usually only indicated in those cases of persistent shoulder pain which have been unresponsive to conservative pain management, in order to exclude calcific tendinitis and other diagnoses which are unrelated to the rotator cuff (European Commission, 2001; RCR 2017). On the other hand, an US scan remains the primary investigation of choice to assess those pathologies which are related to impingement syndrome or a suspected rotator cuff tear, especially in those cases which are unresponsive to first-line treatments and require the administration of US-guided injections (European Commission, 2001; RCR 2017). Furthermore, US can also be used pre-operatively, whenever a surgeon requires an assessment of the rotator cuff's integrity (European Commission, 2001; RCR 2017).

The shoulder has registered the highest number of fully indicated imaging requests ( $55.9 \%$ ) by Maltese G.Ps, amongst all the five body regions that have been investigated in this study (Refer to Figure 6). In the case of trauma, multiple, plain $\mathrm{x}$-ray orthogonal views are required since some shoulder dislocations may present with subtle findings (European Commission, 2001; RCR, 2017). Moreover, MRI is typically considered as an alternative to US in the investigation of major shoulder trauma as it enables the assessment of complex soft tissue injuries and other bony abnormalities (European Commission, 2001; RCR 2017).

CT scanning of the shoulder may also come in handy in the evaluation of complex bony injuries or whenever plain xray investigations have resulted as inconclusive (RCR, 2017). Comparingly, the corresponding number of imaging examinations that have met the clinical requirements for referral as per the national diagnostic imaging pathways in the study by Rawls and Pighills (2018) were $26 \%$ for plain knee x-ray examinations and $46 \%$ for plain shoulder x-ray examinations, respectively.

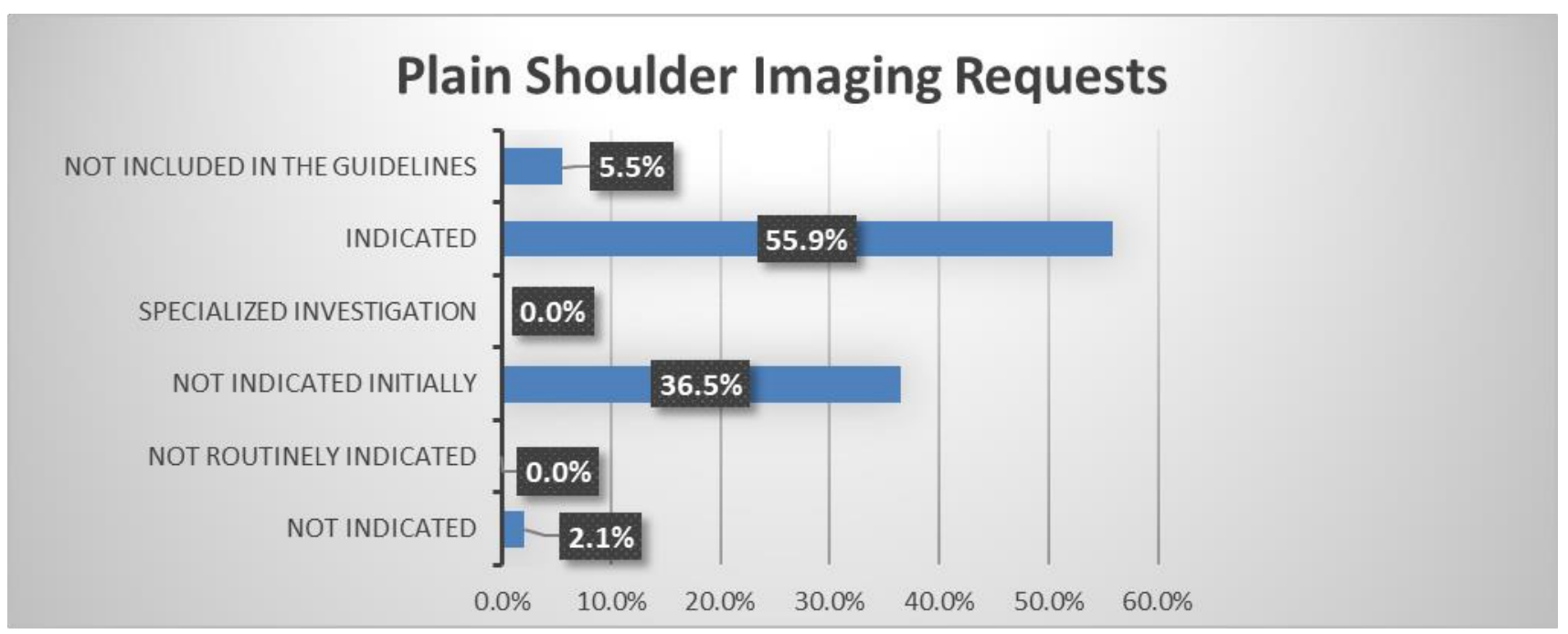

Figure 6 (The indications of the plain shoulder imaging request forms)

\section{1 - Conclusion and recommendations}

It can be concluded that on average, only $20.5 \%$ of plain, musculo-skeletal imaging requests being referred by Maltese G.Ps are classified as indicated for the diagnostic or clinical management purposes. The shoulder is the area which has registered the highest number of clinically indicated requests. In addition, $65.3 \%$ of plain, musculoskeletal imaging requests were classified in the not routinely indicated category. The lumbo-sacral spine is the region that has registered the highest number of cases in the not routinely indicated category; being closely followed by the 


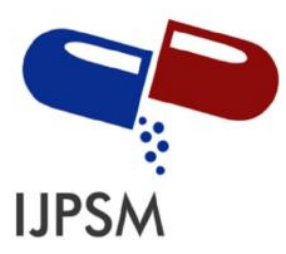

Georgiana Farrugia Bonnici et al, Int. Journal of Pharmaceutical Sciences and Medicine (IJPSM), Vol.6 Issue. 8, August- 2021, pg. 1-16

ISSN: 2519-9889

Impact Factor: 3.426

knee and the cervical spine respectively. These findings indicate that there is a greater tendency for Maltese G.Ps. to request plain, musculoskeletal imaging investigations which are not routinely warranted according to the European Commission (2001) RP 118 and the RCR (2017) i-Refer imaging referral guidelines. Moreover, $40.0 \%$ of these imaging requests were also being referred with insufficient clinical details. The most common types of information that were omitted by the referrers include the patient's age, the joint's level of functional mobility as well as the onset and the duration of any reported musculoskeletal pain.

\subsection{1 - The recommendations based on the research findings and available literature}

\subsubsection{1 - The recommendations for further research}

The replication of this research study on a larger scale would help in furtherly increasing both the generalization and the consistency of the findings and in developing a stronger foundation for the synthesis of higher level of evidence studies. The inclusion of data from private practice G.Ps may also be considered to aid in the comparison of different working groups of Maltese G.Ps. The summarization of the effective radiation doses that are being received by patients as well as the approximate health-care costs related to the inappropriate imaging referrals is also a topic that could be potentially delved into in the near future.

Moreover, an interventional research study which involves the comparison of the imaging referral performance in a representative sample of local G.Ps, before and after the introduction of a national evidence-based implementation strategy which may include monitored multi-disciplinary training, the provision of free educational material, as well as the widespread use of the imaging referral guidelines over a stipulated time-frame would also aid in assessing which performance indicators are most likely to improve the imaging referral processes in Malta by increasing the rates of fully indicated imaging requests. These studies will address the remaining gaps in the literature, as well as provide additional, valuable knowledge about this particular research topic. Ultimately, the conduction of additional valid and reliable research studies will facilitate the adoption of the processes of evidence-based practices and translation sciences.

\subsubsection{2 - The recommendations for continuing medical education and training}

Continuing medical education and training in radiation safety measures and the justification of imaging referral criteria is recommended for Maltese G.Ps. In addition, a health-centre based library containing various related journals and other hard-copies of informative sources is also commendable. Recommended topics for CME updates include the differentiation between the stochastic effects and the deterministic effects of ionizing radiation, an insight into the typical effective radiation doses that are frequently being received by the patients in commonly requested imaging procedures, as well as a briefing about the formal use of European imaging referral guidelines with particular focus on managing non-specific pain in presenting musculoskeletal conditions. It would also be beneficial if such training is formally incorporated as part of the local G.P. training programme curriculum, as it will improve their overall knowledge on these two aforementioned topics and by extension, to their patients, since they are more likely to be properly informed about all radiation-imposed risks. Furthermore, patients are more likely to benefit from a reduction in ionizing radiation exposures from unnecessary imaging investigations.

\subsubsection{3 - The recommendations for improving the primary health-care system clinical practice pathways}

The universal quality improvement strategic levers include the use and the application of measurements and feedbacks, public reporting processes, learning and technical assistances, certifications, accreditations and regulations, incentives and benefits designs, as well as health information technologies (LoBiondo-Wood and Haber, 2017). 


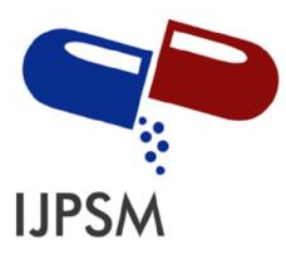

Georgiana Farrugia Bonnici et al, Int. Journal of Pharmaceutical Sciences and Medicine (IJPSM),

Vol.6 Issue. 8, August- 2021, pg. 1-16

ISSN: 2519-9889

Impact Factor: 3.426

Clinical practice in local health centres is likely to be improved via the introduction of any of the below listed recommendations:

- Issuing of regular departmental memos to G.Ps about related evidence-based research findings;

- Ensuring the availability of officially-endorsed imaging referral guidelines on the desktops of all work-place computers;

- Encouraging the use of official imaging referral guidelines, as well as kindling the safety-net of asking for advice from senior colleagues or radiologists, whenever the referrers are in doubt about the management of a particular clinical case;

- Promoting the development of refinements and updates in the locally applicable imaging referral guidelines, with the intention of improving any potential gaps or inconsistencies found in the older versions;

- Considering the introduction of a pro-forma clinical checklist for requesting online plain imaging investigations, to aid G.Ps in improving the appropriateness of their imaging requests at times of clinical controversy, as well as in providing all the relevant clinical details for detailed imaging reporting;

- Organizing a radiation protection campaign to both the Primary Health Care Department (Malta) doctors and to the members of the general public;

- Considering the use of radiation safety education booklets or posters that may be distributed or show-cased to patients whilst they are still in the health-centre waiting areas.

In conclusion, various literature sources point out that the over-use of medical imaging services is becoming universally common. Whilst all patients are entitled to complete diagnoses with sound clinical management plans, these steps should never be at the detriment to their own health or to the extent of excessively increasing the health-care costs or experiencing untimely delays which are attributable to higher workloads of the radiology departments. In fact, the universal six dimensions of health-care quality revolve around safety, effectiveness, efficiency, equity, as well as timely, patient-centred care (LoBiondo-Wood and Haber, 2017). Findings from this research study suggest the need for further education in radiation safety and the imaging referral processes amongst the Maltese G.P. community who also have an important, pivotal role in acting as daily gate-keepers to various specialist treatment care pathways. The use of imaging referral guidelines is strongly suggested, especially at times of clinical difficulty or controversy. Combined together, all of the previously recommended measures for improvement will ensure maximal patient safety from the proper justification of imaging requests via an effective risk-benefit analysis pertaining to ionizing medical imaging procedures.

\section{References}

[1]. Ashurst, J.V., Cherney, A.R., Evans, E.M. (2014). Research priorities for the influence of gender on diagnostic imaging choices in the emergency department setting. Acad Emerg Med 2014;21:1431-7.

[2]. Azman, R.R., Shah, M.Z., Hoong N.K. (2019). Radiation safety in emergency medicine: Balancing the benefits and risks. Korean J Radiol (2019):20(3)399-404.

[3]. Beck, C., Polit, D.F. (2010). Essentials of Nursing Research: Methods, Appraisal \& Utilization. (7 ${ }^{\text {th }}$ edition). Philadelphia: Lippincott, Williams \& Wilkins.

[4]. Brownlee, S., Chalkidou, K., Doust, J, (2017). Evidence for overuse of medical services around the world. Lancet 2017;390:156-68.

[5]. Camilleri, L., (2020). Personal communication with the author at the Faculty of Maths and Statistics, University of Malta, March 9, 2020; as well as the Introduction, Intermediate and Advanced SPSS course notes (2020).

[6]. Creative Research Systems (2020). Sample Size Calculator. Accessed via https://www.surveysystem.com/sscalc.htm on 31 Oct 2020. 


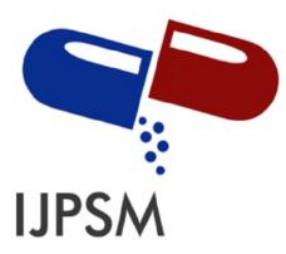

Georgiana Farrugia Bonnici et al, Int. Journal of Pharmaceutical Sciences and Medicine (IJPSM),

Vol.6 Issue. 8, August- 2021, pg. 1-16

ISSN: 2519-9889

Impact Factor: 3.426

[7]. European Commission (2001). Radiation protection 118: referral guidelines for imaging. Luxembourg: Office for Official Publications of the European Communities.

[8]. European Commission (2008) European Commission guidance on estimating population doses from medical $X$-ray procedures (Radiation Protection No 154). Luxemburg: Office for Official Publications of the European Communities; 2008.

[9]. European Commission (2015). Medical radiation exposure of the European population. Radiation Protection 180. Luxembourg: Publications Office of the European Union, 2015. ISBN: 978-92-79-45374-8.

[10].Farrugia, G. (2016). Ionizing radiation is a hazard: Is your imaging request justified? The International Journal of Medical Students, Suppliment Issue (2017).

[11].Grove, S.K., Cipher, J. D., (2019) Statistics for nursing research: A workbook for evidence-based practice. $2^{\text {nd }}$ edition. UK: Elsevier Publishers.

[12].Hamada, N., Fujimichi, Y. (2014). Classification of radiation effects for dose limitation purposes: history, current situation and future prospects. J Radiat Res 2014;55:629-40.

[13].Hill, K.D., Einstein, A., (2016). New approaches to reduce radiation exposure. Trends Cardiovasc Med. 2016 January ; 26(1): 55-65.

[14].Lehnert, B.E., Bree, R.L. (2010). Analysis of appropriateness of outpatient CT and MRI referred from primary care clinics at an academic medical center: how critical is the need for improved decision support? $J$ Am Coll Radiol 2010;7:192-7.

[15].Lim, Y.Z., Chou, L., Rau, T.M. (2019). People with low back pain want clear, consistent and personalized information on prognosis, treatment options, and self-management strategies: A systematic review. Journal of Physiotherapy (65)2019, 124-135.

[16].LoBiondo-Wood, G., Haber, J. (2017). Nursing Research: Methods and critical appraisal for evidence-based practice. $9^{\text {th }}$ edition. UK: Elsevier Publishers.

[17].Rehani, M. M. (2017) "Patient radiation exposure and dose tracking: a perspective," J. Med. Imag. 4(3), 031206, 2017.

[18].Malone, J., O’Connor, U., Faulkner, K. (2009). Ethical and justification issues in medical radiation protection. Radiat Prot Dosimetry Special Issue 2009;135:Issue 2.

[19].Malone, J. (2008) Radiation protection, ethics, law and public awareness: in harmony or out of tune. European Congress of Radiology (ECR) Book of Abstracts. Eur Radiol Suppl 2008;18:128.

[20].Malone, J., Guleria, R., Craven, C. (2012). Justification of diagnostic medical exposures: some practical issues. Report of an International Atomic Energy Agency Consultation. The British Journal of Radiology 85 (2012), 523-538.

[21].Mendelson, R.M., Montgomery, B.D. (2016). Towards appropriate imaging: Tips for practice. The Royal Australian College of General Practitioners 2016. AFP Vol.45, No.6, June 2016.

[22].Miller, J.D., Avedian, R.S., Rajani. R. (2015). What is the use of imaging before referral to an orthopaedic oncologist? A prospective, multi-center investigation. Clinical orthopaedics and related research (2015): 473:868-874.

[23].Pitman, A.G. (2017). Quality of referral: What information should be included in a request for diagnostic imaging when a patient is referred to a clinical radiologist? Journal of Medical Imaging and Radiation Oncology, 61(2017); 299-303.

[24].Pullicino, G., Pavia, J., Zintilis, S., Francalanza, D., Sciortino, P., Sciortino, P. (2016). Imaging of low back pain in a public health centre. A study of test request behaviour of doctors. The Journal of the Malta College of Family Doctors; Volume 05 issue 03 December 2016.

[25].Rawle, M., Pighills, A. (2018) Prevalence of unjustified emergency department x-ray examination referrals performed in a regional Queensland hospital. J Med Radiat Sci 2018; 65: 184-91.

[26]. Royal College of Radiologists (RCR) (2017). iRefer Imaging Referral Guidelines: Making the best use of clinical radiology, 8th edition. London: The Royal College of Radiologists, 2017. Accessed via http://www.rcr.ac.uk/content.aspx?PageID=995 on 28 Dec 2019. 


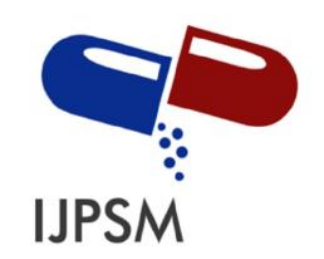

Georgiana Farrugia Bonnici et al, Int. Journal of Pharmaceutical Sciences and Medicine (IJPSM),

Vol.6 Issue. 8, August- 2021, pg. 1-16

ISSN: 2519-9889

Impact Factor: 3.426

[27].Suman, A., Scaafhmsa, F.G., Van de Ven, P.M. (2018). Effectiveness of a multi-faceted implementation strategy compared to usual care on low back pain guideline adherence amongst general practitioners. $B M C$ Health Services Research (2018).

[28].Vilar-Palop, J., Hernandez-Aguado, I., Pastor-Valero, M., Vilar, J., González-Alvarez, I., Lumbreras, B. (2018). Appropriate use of medical imaging in two Spanish public hospitals: a cross-sectional analysis. BMJ Open 2018;8:e019535.

[29].Zontar, D., Zdesar, U., Kuhelj, D., Pekarovic, D., Skrk D. (2015). Estimated collective effective dose to the population from radiological examinations in Slovenia. Radiol Oncol 2015; 49(1): 99-106.

\section{A Brief Author Biography}

Georgiana Farrugia Bonnici, BSC (Hons) Radiography, M.D., MSc Family Medicine - Dr. Georgiana Farrugia Bonnici is a former diagnostic radiographer and a medical doctor. She has completed a Masters of Science in Family Medicine and is a prospective GP trainee. Her research field interests are general practice, cardiology, respiratory medicine and radiology.

Ryan Grech, M.D. - Dr Ryan Grech is a radiology specialist registrar within the NHS Greater Glasgow and Clyde currently reading for a Masters in Imaging with the University of Edinburgh. His research field interests include, radiology and the use of digital interventions including artificial intelligence in healthcare. 